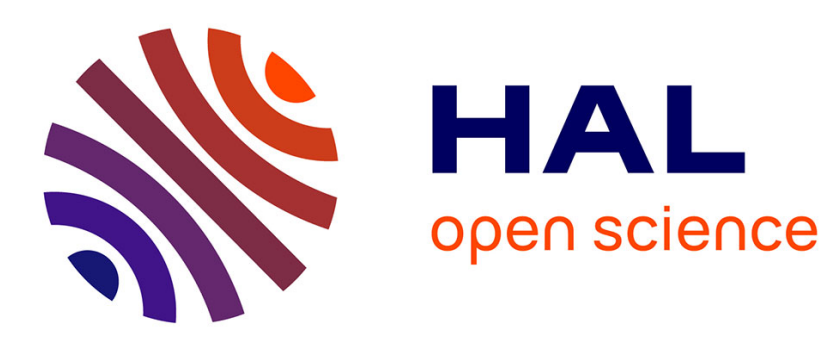

\title{
Spontaneously Flowing Crystal of Self-Propelled Particles
}

\author{
Guillaume Briand, Michael Schindler, Olivier Dauchot
}

\section{To cite this version:}

Guillaume Briand, Michael Schindler, Olivier Dauchot. Spontaneously Flowing Crystal of SelfPropelled Particles. Physical Review Letters, 2018, 120 (20), 10.1103/physrevlett.120.208001 . hal01916201

\section{HAL Id: hal-01916201 \\ https://hal.science/hal-01916201}

Submitted on 8 Nov 2018

HAL is a multi-disciplinary open access archive for the deposit and dissemination of scientific research documents, whether they are published or not. The documents may come from teaching and research institutions in France or abroad, or from public or private research centers.
L'archive ouverte pluridisciplinaire HAL, est destinée au dépôt et à la diffusion de documents scientifiques de niveau recherche, publiés ou non, émanant des établissements d'enseignement et de recherche français ou étrangers, des laboratoires publics ou privés. 


\title{
Spontaneously Flowing Crystal of Self-Propelled Particles
}

\author{
Guillaume Briand, ${ }^{1}$ Michael Schindler, ${ }^{2}$ and Olivier Dauchot ${ }^{1}$ \\ ${ }^{1}$ EC2M, UMR Gulliver 7083 CNRS, ESPCI ParisTech, PSL Research University, 10 rue Vauquelin, 75005 Paris, France \\ ${ }^{2}$ PCT, UMR Gulliver 7083 CNRS, ESPCI ParisTech, PSL Research University, 10 rue Vauquelin, 75005 Paris, France
}

(Received 12 September 2017; revised manuscript received 19 March 2018; published 15 May 2018)

\begin{abstract}
We experimentally and numerically study the structure and dynamics of a monodisperse packing of spontaneously aligning self-propelled hard disks. The packings are such that their equilibrium counterparts form perfectly ordered hexagonal structures. Experimentally, we first form a perfect crystal in a hexagonal arena which respects the same crystalline symmetry. Frustration of the hexagonal order, obtained by removing a few particles, leads to the formation of a rapidly diffusing "droplet." Removing more particles, the whole system spontaneously forms a macroscopic sheared flow, while conserving an overall crystalline structure. This flowing crystalline structure, which we call a "rheocrystal," is made possible by the condensation of shear along localized stacking faults. Numerical simulations very well reproduce the experimental observations and allow us to explore the parameter space. They demonstrate that the rheocrystal is induced neither by frustration nor by noise. They further show that larger systems flow faster while still remaining ordered.
\end{abstract}

DOI: $10.1103 /$ PhysRevLett.120.208001

Crystals, translationally periodic structures, are known for their solid behavior: they sustain their own shape. Liquids are disordered and flow to adopt the shape of their container. Here, we use self-propelled hard disks to show that this common knowledge is undermined in the context of active matter.

In the absence of alignment, the crystallization of selfpropelled disks, with repulsive interactions, follows the two-step scenario reported at equilibrium $[1,2]$. The situation is far less clear when alignment between particles overcomes rotational diffusion. In the fluid phase, polar ordering takes place and collective motion sets in [3-10]. We are interested here in this aligning behavior at high packing fractions, where, in the absence of activity, the equilibrium system forms a crystalline phase.

Within the present state of knowledge, one cannot predict whether the system spontaneously breaks both the rotational symmetry associated with the global phase of the polarity and the translational symmetry of space associated with the structural ordering - a situation analogous to that of supersolidity [11]. On one hand, a mesoscopic field theory [12] predicts a transition from a resting crystal to a traveling crystalline state, where the particles migrate collectively while keeping their crystalline order. On the other hand, simulations [13] of a generalized Vicsek model [14], which accounts for alignment as well as shortranged repulsive interactions, report a mutual exclusion of the polar and the structural order and therefore refute the generality of the theory. Furthermore, in both cases, the speed of the particles and the alignment strength-and equivalently the noise level—are fixed, while, in principle, one expects the density to renormalize these parameters.
Motility induced phase separation, for instance, is precisely a consequence of a decrease in particle speed with increasing local density [15-19].

For any specific system of interest, it is thus of interest to consider the following questions: (i) Does alignment develop at high density, and, if that is the case, does polar order take place? (ii) Is the structural order stable against activity in the presence of polar alignment? For active nematics, activity promotes the proliferation of defects and destroys nematic order [20-24]. (iii) If the traveling crystalline state exists, how does it adapt to confinement? In a fluid phase, active flocks condensate at the boundaries [25] and form vortex flows [26-29]. How does it translate
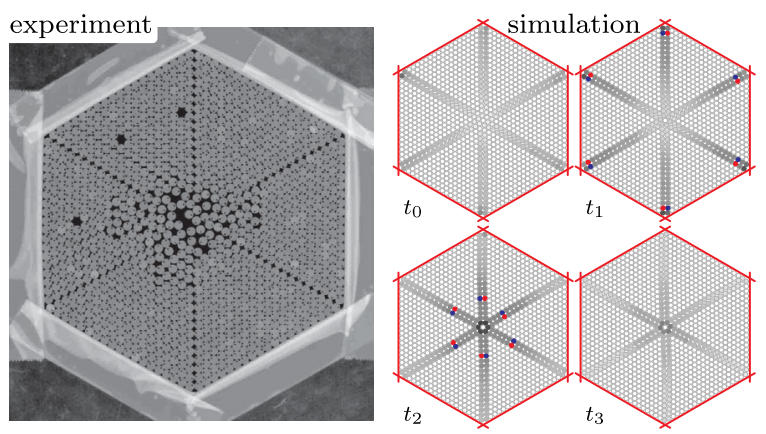

FIG. 1. Flowing crystal configurations in the experiment at a finite noise level with geometrical frustration $(N=1104$, $\phi=0.86$ ), and in simulations at successive times, without noise and without geometrical frustration. The gray levels code for the orientational order parameter; the colors blue and red code for five and seven neighbors, respectively $(N=1141, \phi=0.88)$. 
to a traveling crystalline phase? Does it stop, does it melt, does it fracture?

In this Letter we consider a system of self-propelled hard disks [30], for which alignment emerges from the dynamical relaxation of the particle polarity [31] after each collision and induces collective motion at a moderate packing fraction [7,30,31]. Building upon this well characterized experimental system, we demonstrate (i) that alignment survives at high density, (ii) that the system develops both polar and structural ordering on the experimental length scales, and (iii) that this polar and ordered structure organizes into a coherent sheared flow, which is made possible by the localization of shear along intermittent stacking faults (Fig. 1 and Video SI-1 in the Supplemental Material [32]). The emergence of shear in response to boundary conditions is the basic property defining liquids. For obvious reasons, however, we cannot call this coherently flowing ordered structure a liquid crystal; we shall call it a rheocrystal. The existence of this rheocrystal does not rely on the particular experimental conditions under which it is observed. Simulations very well reproduce the experimentally observed phenomenology (Video SI-2 in the Supplemental Material [32]). Within periodic boundary conditions, they confirm the existence of a traveling ordered structure (Video SI-6). Within the hexagonal geometry, they allow for relaxing the confinement gradually instead of removing particles and varying the control parameters. The structurally ordered flow remains coherent for a large set of parameters and up to the largest scale we could investigate. Finally, the limit of zero noise [Fig. 1 (right) and Video SI-7 in the Supplemental Material [32]] allows us to provide a more precise description of the dynamics along the stacking faults.

The experimental system is made of micro-machined disks (diameter $d=4 \mathrm{~mm}$, total height $2 \mathrm{~mm}$ ), with an off-center tip and a glued rubber skate, located at diametrically opposite positions. These two "legs" with different mechanical responses endow the particles with a polar axis. Subjected to vertical vibration, these polar disks (SPP) perform a persistent random walk, the persistence length of which is set by the vibration parameters (sinusoidal vibration with frequency $f=95 \mathrm{~Hz}$ and amplitude $a$ ) [30]. The maximal acceleration relative to gravity is set to $\Gamma=(2 \pi f)^{2} a / g=2.4$. A glass plate confines the particles from above. We also use plain rotationally invariant disks (same metal, diameter, and height), hereafter called the "isotropic" (ISO) disks. In the present study, the disks are laterally confined in a regular hexagonal arena of side length $79 \mathrm{~mm}$. The motion of all particles is tracked using a standard CCD camera at a frame rate of $30 \mathrm{~Hz}$.

As already stated in Ref. [26], it is possible to fix, but very difficult to fine-tune in a reproducible manner, the selfpropulsion in the above experimental system. This is why, already in Ref. [7], we have constructed a model for the motion and collisions of the polar disks, which accounts quantitatively for the experimental properties at the single and pair interaction level and reproduces very well the macroscopic physics. In this model, later studied in great detail [31], a particle $i$ is described by the position $\mathbf{r}_{i}$ and velocity $\mathbf{v}_{i}$ of its center, and by its body axis given by the unit vector $\hat{\mathbf{n}}_{i}$. Between collisions, these parameters evolve according to the equations

$$
\begin{aligned}
\frac{d}{d t} \mathbf{r}_{i} & =\mathbf{v}_{i}, \\
\tau_{v} \frac{d}{d t} \mathbf{v}_{i} & =\hat{\mathbf{n}}_{i}-\mathbf{v}_{i}, \\
\tau_{n} \frac{d}{d t} \hat{\mathbf{n}}_{i} & =\left(\hat{\mathbf{n}}_{i} \times \hat{\mathbf{v}}_{i}\right) \times \hat{\mathbf{n}}_{i},
\end{aligned}
$$

which have been made dimensionless by choosing suitable units. In Eq. (1), the competition between self-propulsion $\hat{\mathbf{n}}$ and viscous damping $-\mathbf{v}$ lets the velocity relax to $\hat{\mathbf{n}}$ on a timescale $\tau_{v}$. Similarly, in Eq. (1c), the polarity $\hat{\mathbf{n}}$ undergoes an overdamped torque that orients it toward $\mathbf{v}$ on a timescale $\tau_{n}$. Interactions between particles are elastic hard-disk collisions which change $\mathbf{v}$ but not $\hat{\mathbf{n}}$. After such a collision, $\mathbf{v}$ and $\hat{\mathbf{n}}$ are not collinear, and the particles undergo curved trajectories which either are interrupted by another collision, or the particles reach their stationary state, characterized by a straight trajectory at unit speed $\mathbf{v}=\hat{\mathbf{n}}$. The final direction of $\mathbf{v}$ (and of $\hat{\mathbf{n}}$ ) depends on the parameter $\alpha=\tau_{n} / \tau_{v}$, which can be understood as the persistence of the polarity $\hat{\mathbf{n}}$. On top of the deterministic trajectories given by Eq. (1), we add to both vectors a common angular noise, normally distributed with zero mean and variance $2 D$ (see Ref. [31] for implementation details). In the following, unless specified, only the size of the arena and the number of particles are varied; the parameters $\tau_{v}=0.25, \alpha=0.867, D=2.16$ have been chosen so as to be in the experimental range. The hexagonal packing is qualified using the local orientational order parameter $\psi_{6}:=\left|\sum_{q} \exp \left(6 i \theta_{p q}\right)\right| / n_{p}$, defined as a perparticle quantity. Here, the sum runs over the $n_{p}$ neighbors of particle $p$, and $\theta_{p q}$ is the angle of the connecting vector, with respect to a fixed axis. Particle neighborship is defined by Voronoi tessellation.

We start with the description of the experimental results, at the root of the present work. Figure 2 reports some static aspects of the structures obtained in the experiment. The densest hexagonal packing we can form in the arena ( $N=1141, \phi=0.89)$ is first prepared, before we remove particles at random. The defectless structures (leftmost column) are stable, for both the ISO and SPP disks. In the case of the ISO disks (top row), removing a few particles creates defects, which locally lower the individual order parameter $\psi_{6}$ (panels $\left.N=1123,1085\right)$. When the number of removed particles corresponds exactly to the outermost layer of the hexagonal packing (here 114 

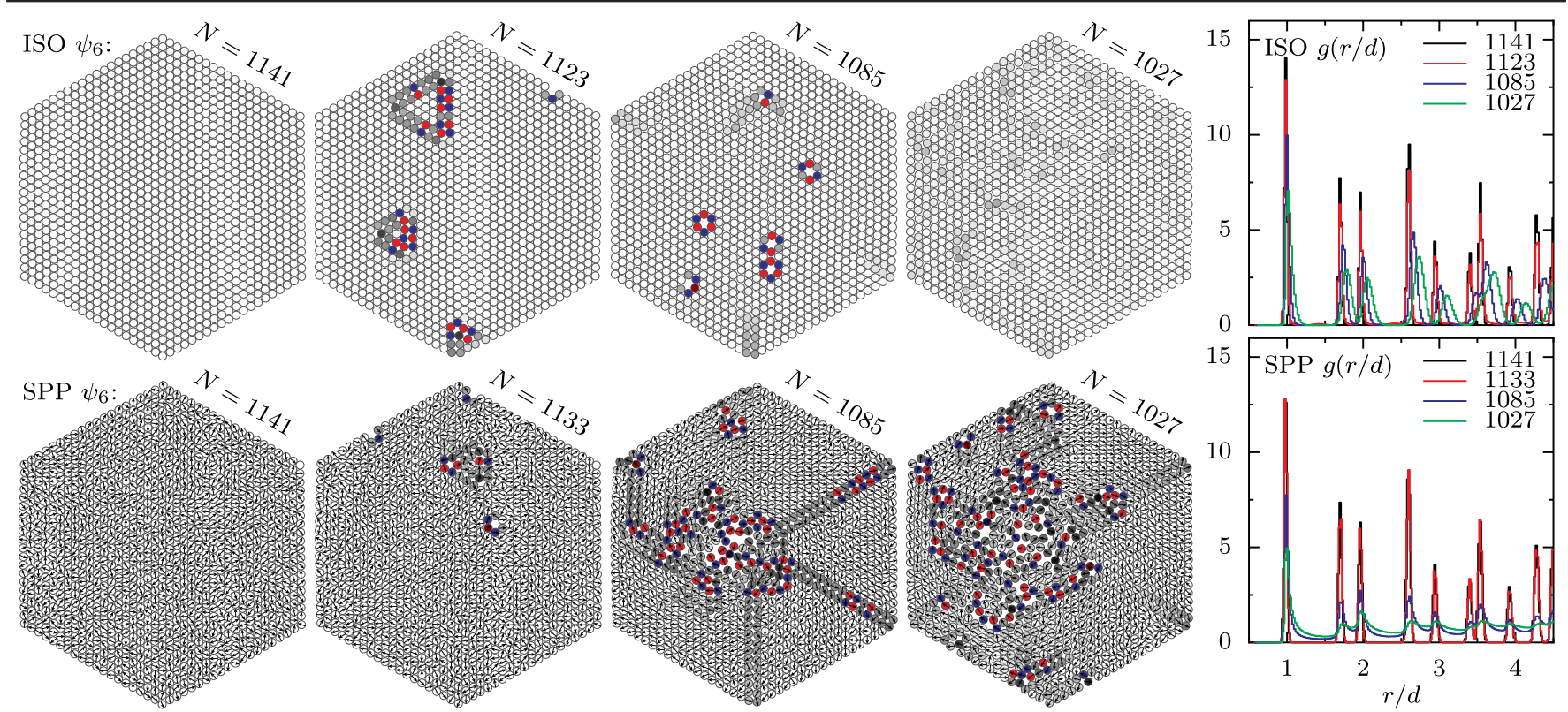

FIG. 2. Experimental data, static aspects: The left panels show time snapshots of isotropic disks (ISO, top row) and of self-propelled polar disks (SPP, bottom row). The gray scale indicates individual values of the local orientational order parameter $\psi_{6} \in[0,1]$, except when there are more or less six Voronoi neighbors (red and blue, respectively). The rightmost panels show the corresponding pair correlation functions, obtained from time averages over trajectories.

particles, passing to $N=1027$ ), one recovers a crystal of ISO disks without geometric frustration and still far from melting $(\phi=0.801)$.

By contrast, in the case of SPP disks (bottom row) the structure becomes increasingly disordered, and defects proliferate. This disorder is reflected in the shape of the pair correlation functions (rightmost column in Fig. 2), which do not drop to zero between peaks. A remarkable feature is the existence of highly ordered sectors, which are separated by less dense pairs of lines where the local symmetry is square $(N=1085)$. One further notices an increasingly large zone of lower density in the center of the arena $(N=1085,1027)$.

The three regimes foreshadowed in the bottom row of Fig. $2(N=1141,1133,1085)$ also differ in their dynamics, as can be seen from Fig. 3 and from Videos SI-3 to SI-5 in the Supplemental Material [32]. For the perfect hexagonal packing, the SPP disks remain trapped at the same average position, and the corresponding mean square displacements (MSDs) are those of a frozen structure (see Video SI-3). For $N=1133$, one observes jumps in the particle trajectories in both the parallel and perpendicular directions [33]: the particles exchange neighbors, change layers, and overall diffuse in both directions, as evidenced by the linear increase with time of their MSDs. Video SI-4 reveals the existence of small but persistent depleted regions, or loose "droplets," which rapidly explore all the system. These droplets are composed of vacancies and defects, which condensate the volume left by the removed particles. Such droplets already are complex objects, which split and merge, the motion of which does not reduce to that of a specific defect. In this regime, we are
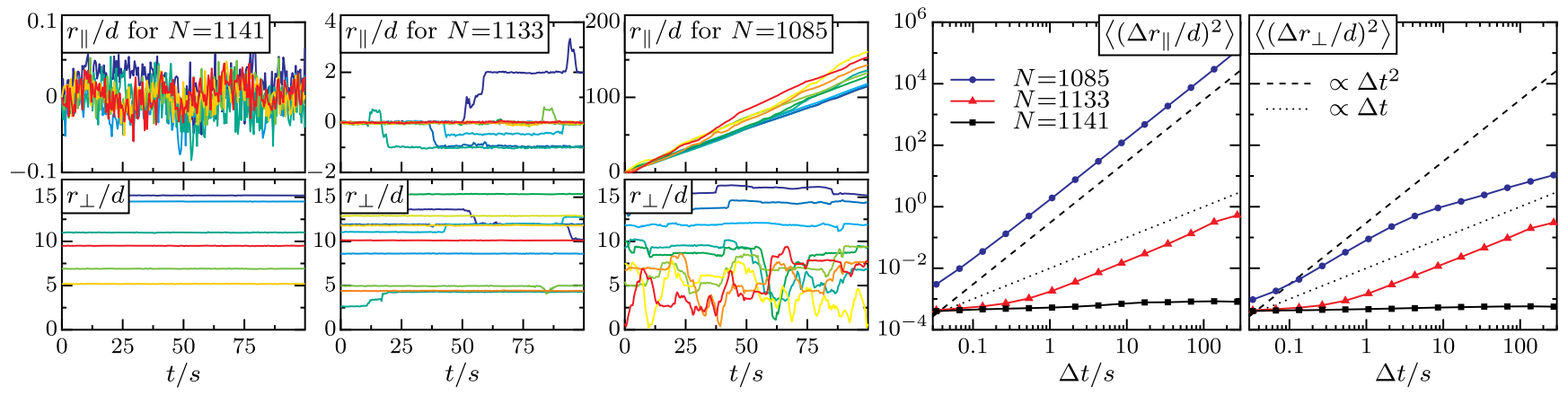

FIG. 3. Experimental data, SPP dynamics: The panels on the left show typical trajectories for different $N$. Plotted are the perpendicular and (unwrapped) parallel components of the particle position [33]. The panels on the right show the corresponding mean squared displacements. 
thus in the presence of a crystalline structure, the density fluctuations of which relax on fast timescales. This finding extends the previously reported decoupling between structure and dynamics [34] to a very large packing fraction. Finally, for $N=1085$, one observes ballistic-like displacement along the direction parallel to the walls, while the perpendicular motion remains diffusive. Video SI-5 shows how the whole crystalline structure starts flowing and forms a global rotation, responsible for the reported ballistic motion. This flowing crystalline structure is made possible by the condensation of shear along localized stacking faults, dividing the hexagon into regular triangles. Along these lines, the local symmetry oscillates between hexagonal and square, as the blocks slide past each other.

The above results clearly answer the questions raised in the Introduction. In particular, they demonstrate that the dynamical alignment survives the high-frequency collisions and triggers collective motions, which take the form of a structurally ordered flow. Accordingly, one expects that, within periodic boundaries, a traveling crystal will form, as we confirm with numerical simulations (see Video SI-6 in the Supplemental Material [32]). They also raise a number of new questions: (i) By removing particles, we induce geometrical frustration. Is this necessary for the global rotation to develop? (ii) While the boundaries facilitate the crystalline order, defects concentrate in the hexagon center, where the rotating flow does not develop. What if the system size is increased? (iii) How do parameters such as density or noise level impact on the rheocrystal? To answer these questions, we now turn to numerical simulations of the model introduced above.

First, the global motion is not induced by geometric frustration. Instead of removing particles, we target the same packing fraction without geometric frustration by increasing the arena area $A$. Figure 4 displays the parallel and perpendicular MSDs for the SPP disks, once obtained by removing particles (top row) and once by increasing $A$ (bottom row). The packing fractions are the same in both rows, but the dynamical behavior is not: the parallel diffusion at $N=1126$ is replaced by rotation at the corresponding $A=1027.23$. Removing frustration actually favors the coherent flow of the crystalline structure. It is the droplet regime, where both MSDs are diffusive, which is absent in the absence of frustration. We further learn that the central region of lowered density and order, which is still present in the absence of frustration, does not result from the coalescence of such droplets. It is rather a consequence of the close packing of the particles along the hexagon boundaries.

Second, as summarized in Fig. 5, the larger the system is, the better defined is its structure and the faster it flows: Larger systems have higher principal peaks in the pair correlation function (left panel). Regarding the dynamics, the right panel of Fig. 5 displays the average parallel displacement per unit time as a function of the rescaled

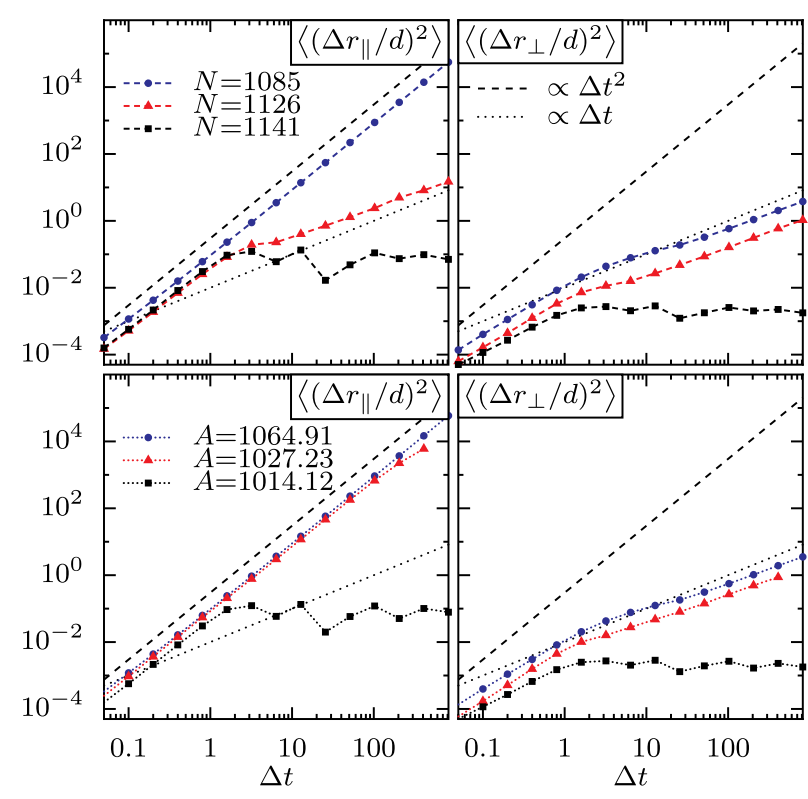

FIG. 4. Simulation data: Variation of $N$ (top row) versus variation of the arena area $A$ (bottom row). Shown are mean squared displacements obtained from pairs $(N, A)$ with the common (bulk) volume fractions $\phi=0.846,0.878$, and 0.890 .

average distance from the center. With increasing size, the rotation becomes more important in two ways: the overall magnitude of the rotation increases, and the central region, where the rotation is reduced, occupies a smaller fraction of the system. In all simulations used for Fig. 5, the vortex flow organizes at the scale of the system size. From that point of view, alignment actually promotes system-size correlations in the structure [35]. The breaking of structure, necessary to allow global flow, happens only along the fault lines, where particles temporarily adopt a local square symmetry. These lines become less important with increasing system size, since they scale as length, not as area.

Third, as shown in Fig. 6, the phase diagram is strikingly similar to the one observed in the limit of vanishing densities [see the curves in Fig. 6 (left), and also
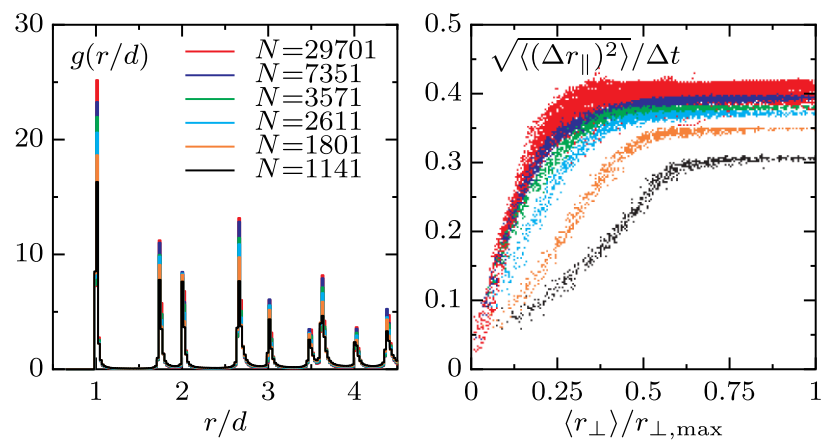

FIG. 5. Simulation data from systems of different sizes. Left: Pair-correlation functions. Right: Average displacement per unit time vs distance from the center rescaled by the system size $(A=1027.23, \phi=0.878, \Delta t=256)$. 


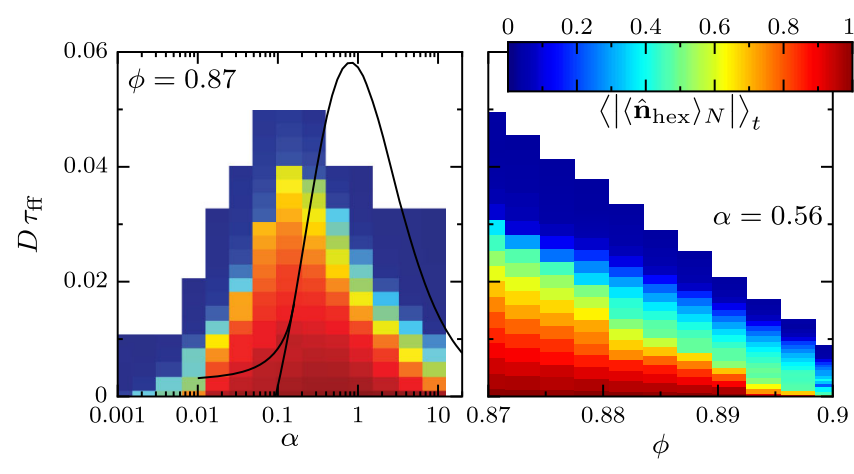

FIG. 6. Simulation data, exploring the parameter space in the $(\alpha, D)$ and $(\phi, D)$ planes. The color codes for coincidence of individual orientation vectors with the orientations of global flow, first averaged over the particles, then over time. Noise levels are scaled by the free-flight time $\tau_{\mathrm{ff}}(\phi)$, as measured at equilibrium. The curves in the left panel are the disorder-polar transition lines in the dilute limit, taken from Ref. [31], but scaled by 0.2 for reasons of visual presentation.

Fig. 2(c) in Ref. [31]]. For strong noise, no collective motion takes place and a static, but active, crystal is stabilized. For weak noise, the rheocrystal occupies a significant range of $\alpha$. As in the dilute case [curves in Fig. 6 (left)], the flowing region has a peaklike shape; for moderate noise, too small or too large polarity persistence hinders collective alignment. Note the nontrivial influence of the density: on the large- $\alpha$ side of the peak, density promotes disorder [Fig. 6 (right)], while it contributes to alignment on the low- $\alpha$ side.

Finally, taking advantage of the fact that the rheocrystal is present in the absence of noise, we clarify the dynamical mechanisms taking place along the stacking fault lines (see Video SI-7 in the Supplemental Material [32]). The lattices in the different sectors of the hexagon match seamlessly only at specific times (Fig. 1 at $t_{3}$ ). At most times, the active flow distorts the structure, very locally, along the stacking fault lines. This starts with the emission of dislocations from the boundary corners. They rapidly travel towards the center, where they merge and vanish. Note, however, that the mismatch across these fault lines is a displacement of the hexagonal lattice, not a rotation. We therefore do not think of them as grain boundaries.

Altogether, the present experimental work, supported by numerical simulations, has unveiled a new behavior of active matter, which had not been observed before. Let us conclude by stressing that many open questions remain, especially regarding the precise nature of the traveling crystal phase, in the large- $N$ limit. Further intensive numerical simulations will be necessary to address these issues.

We acknowledge financial support from Ecole Doctorale ED564 "Physique en Ile de France" for Guillaume Briand's Ph.D. grant. We thank Vincenzo Vitelli and Pierre Ronceray for interesting discussions.
[1] J. Bialké, T. Speck, and H. Löwen, Phys. Rev. Lett. 108, 168301 (2012).

[2] J. U. Klamser, S. C. Kapfer, and W. Krauth, arXiv: $1802.10021 \mathrm{v} 1$.

[3] T. Vicsek, A. Czirók, E. Ben-Jacob, I. Cohen, and O. Shochet, Phys. Rev. Lett. 75, 1226 (1995).

[4] J. Toner and Y. Tu, Phys. Rev. Lett. 75, 4326 (1995).

[5] G. Grégoire, H. Chaté, and Y. Tu, Physica (Amsterdam) 181D, 157 (2003).

[6] H. Chaté, F. Ginelli, G. Grégoire, and F. Raynaud, Phys. Rev. E 77, 046113 (2008).

[7] C. A. Weber, T. Hanke, J. Deseigne, S. Léonard, O. Dauchot, E. Frey, and H. Chaté, Phys. Rev. Lett. 110, 208001 (2013).

[8] A. Bricard, J.-B. Caussin, N. Desreumaux, O. Dauchot, and D. Bartolo, Nature (London) 503, 95 (2013).

[9] M. C. Marchetti, J.-F. Joanny, S. Ramaswamy, T. B. Liverpool, J. Prost, M. Rao, and R. A. Simha, Rev. Mod. Phys. 85, 1143 (2013).

[10] A. Peshkov, E. M. Bertin, F. Ginelli, and H. Chaté, Eur. Phys. J. Spec. Top. 223, 1315 (2014).

[11] A. Kuklov, N. Prokof'ev, and B. Svistunov, Physics 4, 109 (2011).

[12] A. M. Menzel and H. Löwen, Phys. Rev. Lett. 110, 055702 (2013).

[13] C. A. Weber, C. Bock, and E. Frey, Phys. Rev. Lett. 112, 168301 (2014).

[14] G. Grégoire and H. Chaté, Phys. Rev. Lett. 92, 025702 (2004).

[15] F. D. C. Farrell, M. C. Marchetti, D. Marenduzzo, and J. Tailleur, Phys. Rev. Lett. 108, 248101 (2012).

[16] G. S. Redner, M. F. Hagan, and A. Baskaran, Phys. Rev. Lett. 110, 055701 (2013).

[17] M. E. Cates and J. Tailleur, Annu. Rev. Condens. Matter Phys. 6, 219 (2015).

[18] J. Barre, R. Chetrite, M. Muratori, and F. Peruani, J. Stat. Phys. 158, 589 (2015).

[19] A. Martín-Gómez, D. Levis, A. Díaz-Guilera, and I. Pagonabarraga, arXiv:1801.01002v2.

[20] V. Narayan, S. Ramaswamy, and N. Menon, Science 317, 105 (2007).

[21] T. Sanchez, D. T. N. Chen, S. J. DeCamp, M. Heymann, and Z. Dogic, Nature (London) 491, 431 (2012).

[22] H. H. Wensink, J. Dunkel, S. Heidenreich, K. Drescher, R. E. Goldstein, H. Löwen, and J. M. Yeomans, Proc. Natl. Acad. Sci. U.S.A. 109, 14308 (2012).

[23] L. Giomi, M. J. Bowick, X. Ma, and M. C. Marchetti, Phys. Rev. Lett. 110, 228101 (2013).

[24] S. Zhou, A. Sokolov, O. D. Lavrentovich, and I. S. Aranson, Proc. Natl. Acad. Sci. U.S.A. 111, 1265 (2014).

[25] Y. Fily, A. Baskaran, and M. F. Hagan, Soft Matter 10, 5609 (2014).

[26] J. Deseigne, S. Léonard, O. Dauchot, and H. Chaté, Soft Matter 8, 5629 (2012).

[27] F. G. Woodhouse and R. E. Goldstein, arXiv:1207.5349v1.

[28] A. Bricard, J.-B. Caussin, D. Das, C. Savoie, V. Chikkadi, K. Shitara, O. Chepizhko, F. Peruani, D. Saintillan, and D. Bartolo, Nat. Commun. 6, 7470 (2015).

[29] T. Hiraoka, T. Shimada, and N. Ito, J. Phys. Conf. Ser. 921, 012006 (2017). 
[30] J. Deseigne, O. Dauchot, and H. Chaté, Phys. Rev. Lett. 105, 098001 (2010).

[31] K.-D. Nguyen Thu Lam, M. Schindler, and O. Dauchot, New J. Phys. 17, 113056 (2015).

[32] See Supplemental Material at http://link.aps.org/ supplemental/10.1103/PhysRevLett.120.208001 for further visual evidences of our main observations with seven videos.

[33] We used six different Cartesian coordinate systems in the six sectors of the hexagon, aligned with the respective boundaries. This allows us to speak of parallel and perpendicular coordinates, $r_{\|}, r_{\perp}$.

[34] G. Briand and O. Dauchot, Phys. Rev. Lett. 117, 098004 (2016).

[35] Evidently, the presence of the boundaries dominates the global properties of flow and structure. This may be different for much larger systems, but this is pure speculation. We focus on finite systems, where the importance of boundaries even helps to keep the corresponding equilibrium system crystalline. 